\title{
L'abito non fa il monaco
}

\author{
S. Michelassi ${ }^{1}$, S. Nigrelli ${ }^{1}$, M. Amidone ${ }^{1}$, S. Lo Caputo $^{2}$, G. Tinacci ${ }^{3}$
}

UU.OO. di ${ }^{1}$ Nefrologia e Dialisi

${ }^{2}$ Malattie Infettive

${ }^{3}$ Servizio di Anatomia Patologica, Ospedale S.M. Annunziata, Bagno a Ripoli, Azienda Sanitaria di Firenze, Firenze

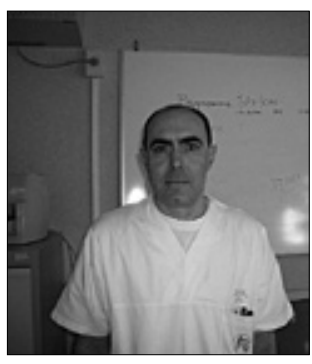

Stefano Michelassi

\section{Caso clinico}

Il signor M. è un paziente maschio di 73 anni di razza caucasica costretto in carrozzina per esiti poliomielitici e per esiti di pregresse fratture traumatiche degli arti inferiori. Ha limitazione funzionale anche degli arti superiori per una lesione bilaterale completa della cuffia dei rotatori del cingolo scapolo-omerale. In passato ha sofferto per nefrolitiasi recidivante ma non è mai stato sottoposto ad interventi invasivi sulle vie urinarie. Nel 2005 (a 69 anni) esami occasionali avevano evidenziato leucopenia che non era stata indagata. La funzione renale e gli altri parametri emocromocitometrici erano risultati nei limiti (per tutti gli esami vedi Tabella I).

Nel 2007 esami di controllo avevano documentato anche lievi anemia e piastrinopenia e segni di nefropatia (proteinuria $100 \mathrm{mg} / \mathrm{dL}$, lieve aumento della creatininemia).

Nei primi mesi del 2008 veniva riscontrata ipertensione arteriosa severa $(210 / 100 \mathrm{mmHg})$, trattata con ACE-inibitore, beta-bloccante, antagonista recettoriale dell'angiotensina II ed alfa-1-litico. L'ecografia addome mostrava reni di dimensioni regolari ma con corticale iperecogena e renella nel gruppo caliceale inferiore sinistro, iperplasia prostatica con vescica da sforzo e residuo postminzionale stimato circa $120 \mathrm{~mL}$. Gli esami evidenziavano peggioramento della funzione renale con proteinuria in range nefrosico, infezione urinaria asintomatica da Enterobacter faecium e grave anemia normocitica normocromica, per cui nel mese di luglio del 2008 il paziente si ricoverava in Nefrologia.

L'esame obiettivo all'ingresso era sostanzialmente negativo, eccetto che per gli esiti poliomielitici e per la presenza di modesti edemi declivi. PA 140/90 mmHg. Gli esami di laboratorio rivelavano anche ipergammaglobulinemia policlonale con piccolo picco monoclonale IgM-k ed immunofissazione urinaria negativa, ipocomplementemia (C3 francamente ridotto, $\mathrm{C} 4$ ai limiti inferiori della norma), positività di ANA e crioglobuline a basso titolo, aumento della VES. L'urinocoltura era positiva per $M y$ cobacterium gordonae, interpretato come contaminante e quindi non trattato.

Rx torace. Iperdiafania dei campi polmonari. Nodulo fibrocalcifico subcentimetrico in campo polmonare superiore sinistro. Minimo versamento pleurico bilaterale. Stria fibrosa basale sinistra.

Ecocardiogramma. Lieve ipertrofia ventricolare sinistra, lieve insufficienza valvolare mitro-aorto-tricuspidalica (PAPS $32 \mathrm{mmHg}$ ), modesto scollamento pericardico (4 mm) circonferenziale.

TC diretta toraco-addominale. Linfonodi mediastinici precarenali di diametro $17-18 \mathrm{~mm}$. Linfonodi subcentimetrici in sede lomboaortica superiore. Minima litiasi caliceale inferiore rene sinistro, cisti renale destra semplice.

Biopsia renale. M.O. (Figg. 1, 2) - 15 glomeruli di cui 5 in sclerosi globale; a carico dei rimanenti diffusa proliferazione cellulare ed espansione della matrice mesangiale con aspetti di lobulazione e interposizione a livello delle pareti capillari. Fibrosi intimale intensa a carico delle arterie, moderata a carico delle arteriole. Aree di atrofia tubulare associata a fibrosi ed infiltrazione linfocitaria nell'interstizio. Gocce ialine nelle cellule epiteliali tubulari, cilindri ialini nei lumi. I.F.: Depositi granulari parietali diffusi di IgG (+++), C3 (+++) e C1q (+); depositi granulari parietali e mesangiali a distribuzione focale $\mathrm{e}$ segmentaria di IgM (+). M.E.: Confermati i dati della microscopia ottica. Depositi elettrondensi in sede mesangiale e subendoteliale. 


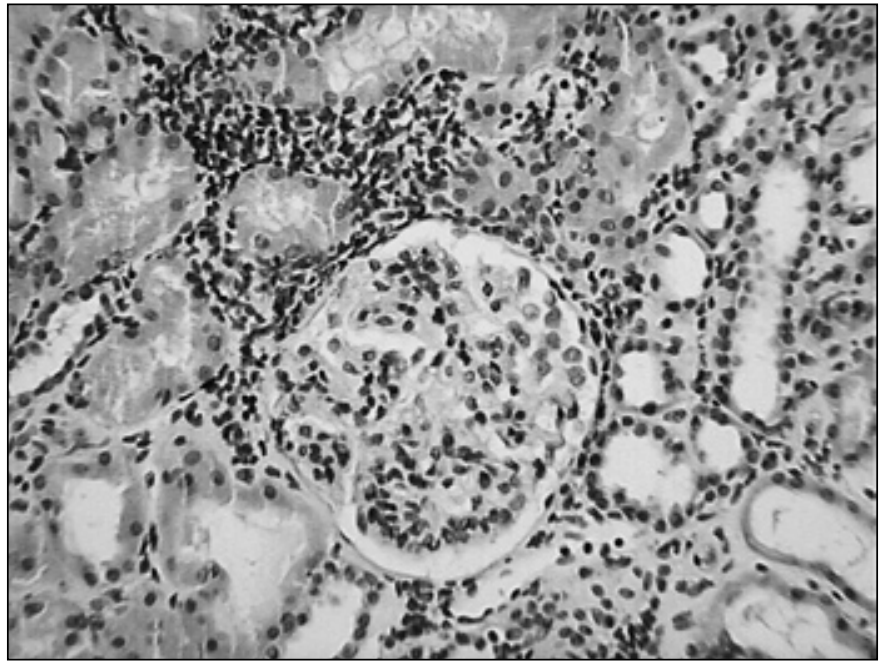

Fig 1 - Ematossilina eosina. Intenso infiltrato interstiziale. A livello glomerulare ipercellularità ed espansione della matrice mesangiale.

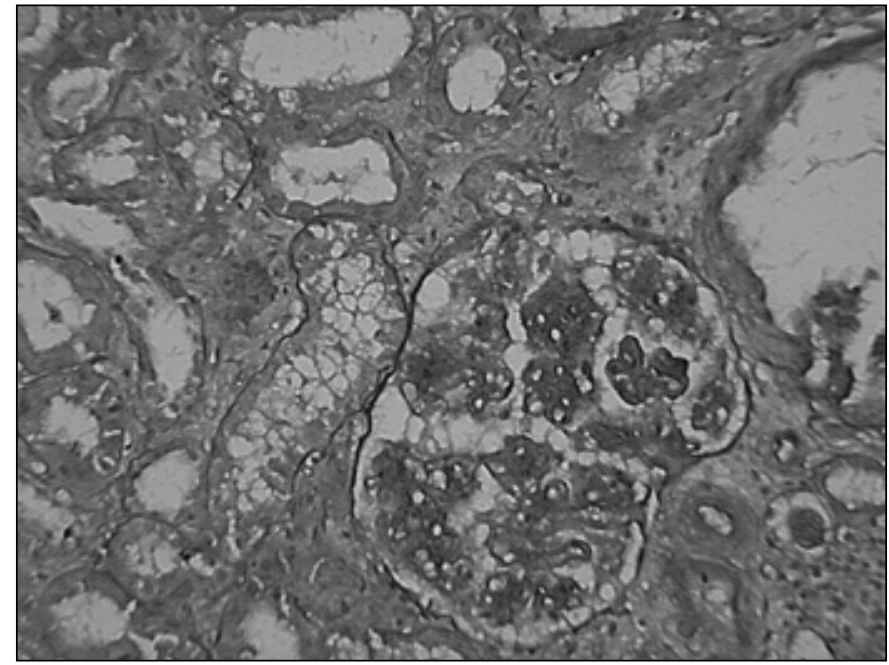

Fig 2 - PAS. Espansione della matrice mesangiale e aspetto lobulare del glomerulo. Ispessimento delle pareti capillari con aspetti di interposizione mesangiale a livello della GBM (aspetto “a doppio contorno”).

TABELLA I - ANDAMENTO ESAMI EMATOURINARI

\begin{tabular}{|c|c|c|c|c|c|c|}
\hline & 2005 & 2007 & $\begin{array}{l}\text { Luglio } \\
2008 \text { * }\end{array}$ & $\begin{array}{c}\text { Ottobre } \\
2008\end{array}$ & $\begin{array}{c}\text { Gennaio } \\
2009\end{array}$ & $\begin{array}{c}\text { Febbraio } \\
2009\end{array}$ \\
\hline Creatinina $(\mathrm{mg} / \mathrm{dL})$ & 0.86 & 1.01 & 1.40 & 1.38 & 2.53 & 3.37 \\
\hline $\mathrm{Hb}(\mathrm{g} / \mathrm{dL})$ & 14.6 & 12.0 & 9.3 & 11.0 & 8.7 & 11.4 \\
\hline $\mathrm{GB}(\mathrm{n} / \mathrm{mcL})$ & 2500 & 3300 & 3000 & 4800 & 3800 & 4000 \\
\hline $\mathrm{N}(\%)$ & & & 47 & 42 & 42 & 66 \\
\hline E (\%) & & & 9 & 7 & 6 & 0 \\
\hline $\mathrm{L}(\%)$ & & & 33 & 38 & 39 & 22 \\
\hline M (\%) & & & 11 & 12 & 11 & 13 \\
\hline Piastrine (n/mcL) & 169000 & 133000 & 110000 & 169000 & 98000 & 10000 \\
\hline Proteine tot $(\mathrm{g} / \mathrm{dL})$ & & & 7.0 & 6.5 & 6.0 & 5.3 \\
\hline Albumina (\%) & & & 38 & 43 & 34 & 35 \\
\hline Gamma-glob (\%) & & & 39 & 26 & 38 & 33 \\
\hline $\operatorname{IgA}(\mathrm{mg} / \mathrm{dL})$ & & & 472 & 389 & & \\
\hline $\operatorname{IgM}(\mathrm{mg} / \mathrm{dL})$ & & & 384 & 206 & & \\
\hline $\operatorname{IgG}(\mathrm{mg} / \mathrm{dL})$ & & & 2460 & 1440 & & \\
\hline Microematuria (semiquant) & & & ++ & ++ & ++ & \\
\hline Micropiuria (semiquant) & & & + & + & + & \\
\hline Proteinuria spot (mg\%) & & 100 & 250 & 100 & 300 & \\
\hline Proteinuria 24 ore $(\mathrm{g} / \mathrm{die})$ & & & 4.3 & 1.5 & 2.2 & \\
\hline Urinocoltura & & & $\begin{array}{l}\text { Mycob. } \\
\text { gordonae }\end{array}$ & NEG & $\begin{array}{l}\text { Enteroc. } \\
\text { faecalis }\end{array}$ & \\
\hline $\mathrm{C} 3(\mathrm{mg} / \mathrm{dL})$ & & & 46 & 83 & 48 & 51 \\
\hline $\mathrm{C} 4(\mathrm{mg} / \mathrm{dl})$ & & & 17 & 27 & 16 & 15 \\
\hline IF siero (monoclon) & & & IgM-k & IgM-k & & \\
\hline IF urine (monoclon) & & & NEG & & & \\
\hline ANA (EIA/IF) & & & $1.1(1: 80)$ & $0.64(1: 80)$ & & \\
\hline Criocrito (\%) & & & 1.0 & & & \\
\hline Coprocoltura & & & & & & $\begin{array}{l}\text { Cl.difficile } \\
\text { (citotox) }\end{array}$ \\
\hline
\end{tabular}

* Altri esami normali o negativi: urinocoltura per germi comuni, Mantoux, enzimi e virus (HCV-RNA, HBV-DNA) epatici, markers neoplastici, PCR, ATC antiDNA, ENA, ATC antifosfolipidi, CIC, test di Coombs, aptoglobina, LDH, RA test, WR, saturazione transferrina e ferritina. 


\section{TEST DI VERIFICA - 4}

Quale è il tipo di glomerulonefrite di cui è affetto il paziente?

1) Glomerulonefrite membranosa (GM)

2) Glomerulonefrite membrano-proliferativa (GNMP)tipo 1

3) Glomerulonefrite acuta postinfettiva

Di questo tipo di glomerulonefrite sono di più frequente riscontro la forma idiopatica o le forme secondarie?

1) La forma idiopatica

2) Le forme secondarie

3) Entrambe hanno pressoché la medesima prevalenza.

Le risposte corrette alle domande sono a pag. 26

Biopsia osteo-midollare. Midollo normorappresentato in relazione all'età del paziente. Plasmocitosi (15 $\%)$ interstiziale e perivascolare, talora in microaggregati, senza atipie citologiche e documentabile restrizione clonale. Macroaggregato nodulare interstiziale costituito da linfociti (CD3++, CD20+) e plasmacellule con caratteristiche di reattività.

L'ematologo diagnosticava MGUS IgM, insufficienza renale cronica secondaria a crioglobulinemia e consigliava terapia steroidea o rituximab.

Il paziente iniziava trattamento con prednisone orale $50 \mathrm{mg} / \mathrm{die}$, associato ad ACE-inibitore, antagonista recettoriale dell'angiotensina II e diuretico, che si associava a riduzione della proteinuria ed a miglioramento del quadro ematologico ed elettroforetico. Tuttavia alla fine di ottobre 2008 la terapia steroidea veniva sospesa per comparsa di diabete mellito, allucinazioni e peggioramento dell'ipertensione arteriosa.

In gennaio e in febbraio 2009 il paziente veniva ricoverato altre due volte nel nostro reparto per peggioramento progressivo delle condizioni generali, comparsa di sintomatologia gastrointestinale (vomito, grave e persistente diarrea, febbricola $37,5{ }^{\circ} \mathrm{C}$ ), neurologica (disartria, episodi di confusione mentale e disorientamento spazio-temporale, progressivo rallentamento psico-motorio) e respiratoria (saltuaria dispnea), ipertensione non controllata, aumento degli edemi declivi.

Gli esami mostravano peggioramento della funzione renale, dell'ipergammaglobulinemia e della pancitopenia, ipocomplementemia persistente. In particolare si assisteva a rapida progressione della piastrinopenia con ATC antipiastrine negativi e senza introduzione di nuovi farmaci. L'urinocoltura risultava positiva per Enterococcus faecalis, negativa per micobatteri. Le coprocolture per Salmonella, Shigella, Campylobacter, cisti protozoarie, uova e larve di elminti erano negative. Risultava invece positiva la ricerca della citotossicità fecale per Clostridium difficile.

Rx torace. Versamento pleurico bilaterale.

Ecografia addominale. Ascite diffusa.

Colonscopia. Mucosa del colon sinistro intensamente eritemato-edematosa, con erosioni fibrinose e aree di aspetto atrofico. Istologicamente, flogosi aspecifica.

TC cranio diretta. Tenue ipodensità sottocorticale occipitale sinistra, modico ampliamento del sistema ventricolare e degli spazi liquorali subaracnoidei.

TABELLA II - FENOTIPIZZAZIONE LINFOCITARIA

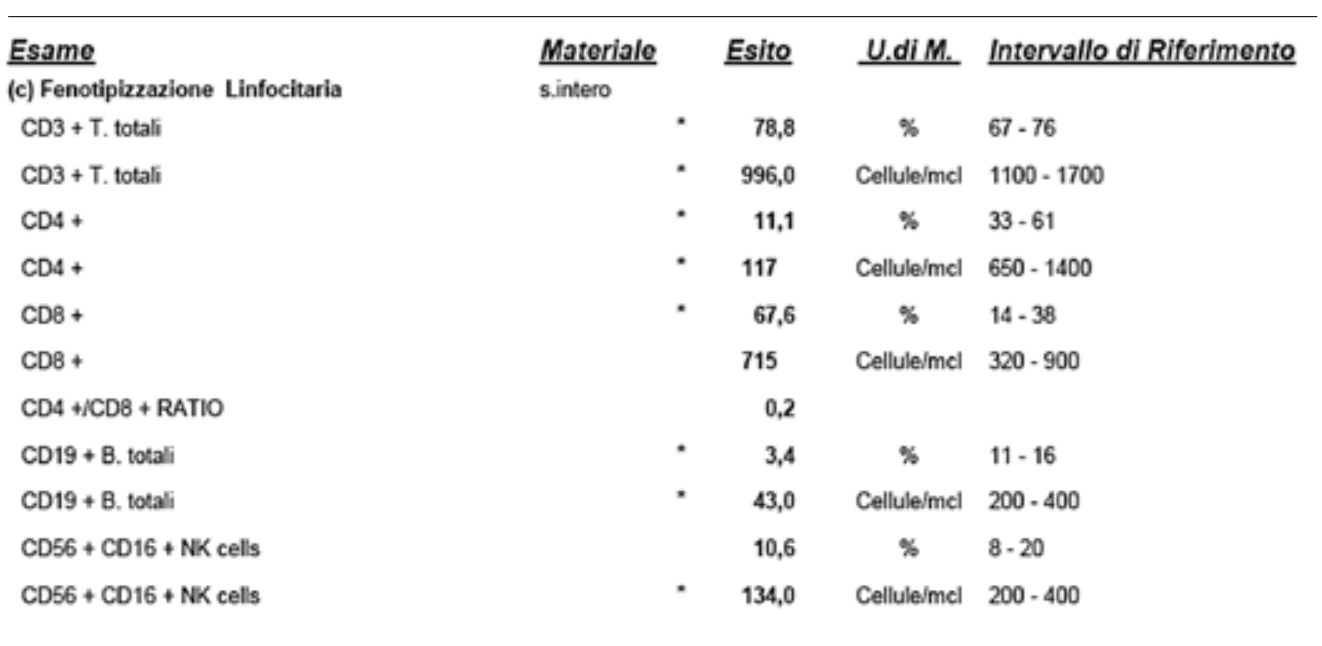




\section{Procedura diagnostica}

Non potendo escludere una forma linfoproliferativa alla base della glomerulonefrite e del complesso quadro clinico, e anche nella prospettiva di intraprendere una terapia con immunosoppressore, veniva eseguita tipizzazione linfocitaria che documentava deplezione di CD4 (117 vs v.n. $650-1400 / \mathrm{mcL}$ ) e inversione del rapporto $\mathrm{CD} 4 / \mathrm{CD} 8$ (Tab. II).

La procedura diagnostica consisteva pertanto nel test per ATC anti-HIV che risultava positivo.

Da una indagine nella cerchia dei familiari sui possibili fattori di rischio per HIV emergeva che in passato il sig. M. aveva tenuto comportamenti sessuali a rischio praticando rapporti sessuali non protetti con partner diverse.

Nei giorni successivi risultavano positive la ricerca di ATG criptococcico e la PCR per citomegalovirus nel sangue del paziente a conferma di una infezione già evoluta ad AIDS (stadio C3, classificazione CDC).

\section{TEST DI VERIFICA - 5}

\section{Quali sono le nefropatie correlabili a infezione da HIV?}

1) Solo la glomerulosclerosi focale segmentale

2) La glomerulosclerosi focale collassante e la glomerulonefrite membranosa

3) Numerosi tipi di lesioni glomerulari, tubulo-interstiziali e dei piccoli vasi

Le risposte corrette alle domande sono a pag. 26

\section{Diagnosi}

Glomerulonefrite membrano-proliferativa tipo 1 verosimilmente secondaria a infezione da HIV in paziente affetto da AIDS (stadio C3).

\section{Discussione}

È ampiamente prevedibile che il paziente nefropatico HIV positivo divenga una figura sempre più frequente nella pratica clinica del nefrologo. Dalla diffusione dell'HAART (highly active antiretroviral therapy) nella seconda metà degli anni Novanta la mortalità si è ridotta in modo significativo, i pazienti vivono più a lungo e hanno più tempo per sviluppare complicanze croniche, fra cui quelle renali.

La gamma di nefropatie associate ad HIV è ampia e soprattutto nei pazienti di razza non nera sembrano prevalere in modo significativo le forme "non-HIVAN". Il nostro paziente, di razza caucasica, aveva una GNMP tipo I complicante una infezione da HIV avanzata sebbene misconosciuta.

La GNMP idiopatica è molto rara. Il signor $\mathrm{M}$. non aveva sospetti delle malattie che più comunemente causano una GNMP tipo 1 secondaria: era $\mathrm{HCV}$ e HBV negativo, non era portatore di shunt ventricolo-atriale, non aveva segni riferibili ad endocardite subacuta o a LES. Erano documentabili crioglobuline circolanti e un quadro elettroforetico sierico caratterizzato da IgM monoclonale ed $\mathrm{IgG}$ policlonale ma con RA test negativo (quindi senza attività IgM anti-IgG). Non erano presenti segni clinici (artralgie/artrite, lesioni cutanee purpuriche/vasculitiche, neuropatie periferiche) né istologici renali (trombi ialini) attribuibili a crioglobulinemia. $\mathrm{La}$ BOM era negativa, non erano presenti linfadenopatie sicuramente patologiche né splenomegalia che potessero indurre sospetto di malignità ematologiche.

Quadri di GN membranoproliferativa tipo I associati a positività per HIV sono stati descritti prevalentemente in pazienti co-infetti da $\mathrm{HCV}$ o in presenza di crioglobulinemia mista (che in tali casi può essere secondaria sia all'HCV sia allo stesso HIV) (6), ma sporadicamente anche indipendentemente da tali co-morbidità $(7,8)$.

Non è possibile escludere con certezza che il sig. M. avesse una forma "idiopatica" casualmente insorta in soggetto HIV positivo ma il peggioramento della funzione renale contemporaneo all'esplosione dell'AIDS e la rarità attuale delle GNMP idiopatiche rendono assai verosimile che la GNMP tipo 1 del nostro paziente fosse correlata allinfezione da HIV. La stessa presenza di depositi di C1q allimmunofluorescenza renale, più frequente nelle forme secondarie, spinge verso questa ipotesi. Infine, come nel nostro caso, altri AA hanno già riportato con steroidi ed ACE-inibitori una riduzione significativa della proteinuria secondaria a GNMP tipo 1 associata ad infezione da HIV avanzata $(\mathrm{CD} 4<20 \mathrm{mcL})$, senza co-infezione $\mathrm{HCV}$, prima di qualsiasi terapia antivirale (8).

Questo caso dimostra come l'interessamento renale nell'infezione da HIV possa avvenire in assenza di chiare manifestazioni cliniche di AIDS. La pancitopenia, l'ipergammaglobulinemia policlonale, il quadro neurologico (associato ad espansione degli spazi liquorali), il quadro gastrointestinale erano tutte possibili manifestazioni di HIV/AIDS, ma anche tutte assolutamente aspecifiche. Inoltre, l'età e l'aspetto del sig. M. non lo 
etichettavano certo come paziente a rischio Ciò significa che dobbiamo imparare a stare all'erta. In un'epoca in cui l'HAART sta rendendo cronica una malattia in passato molto più rapida, le manifestazioni dell'AIDS possono diventare ancora più subdole e proteiformi, e quando il nefrologo si trova dinanzi ad una nefropatia non chiara, non deve scordarsi di includere l'HIV nella sua diagnosi differenziale.

\section{Riassunto}

Il caso clinico riguarda un paziente anziano con anamnesi pregressa non significativa che si presenta in ambiente nefrologico con un quadro nefritico associato a progressiva pancitopenia. Gli accertamenti documenteranno una condizione patologica che, diversamente dal passato, rischia di diventare sempre più frequente nella pratica clinica del nefrologo.

Parole chiave: Glomerulonefrite, Pancitopenia, Ipocomplementemia

\section{Indirizzo degli Autori:}

Stefano Michelassi, MD

UU.OO. di Nefrologia e Dialisi

Ospedale S.M. Annunziata

Bagno a Ripoli

Azienda Sanitaria di Firenze

Firenze, Italy

smichelassi@tin.it

\section{Bibliografia}

1. Rose BD. Causes of membranoproliferative glomerulonephritis. www.uptodate.com.

2. Rao TK, Filippone EJ, Nicastri AD, et al. Associated FSGS in AIDS. N Engl J Med 1984; 310: 669.

3. D'Agati V, Suh JI, Carbone L, et al. Pathology of HIVassociated nephropathy. Kidney Int 1989; 35: 1358.

4. Rose BD, Appel GB. Collapsing FGS and other renal diseases associated with HIV infection. www.uptodate.com.

5. Haas M, Kaul S, Eustace JA. HIV-associated immune complex glomerulonephritis with 'lupus-like' features: A clinicopathologic study of 14 cases. Kid Int 2005; 67: 1381.

6. Szczech LA, Gupta SK, Habash R, et al. The clinical epidemiology and course of the spectrum of renal diseases associated with HIV infection. Kidney Int 2004; 66: 1145.

7. Chidambaram M, Stigant CE, Sugar LM, et al. Type I membranoproliferative glomerulonephritis in an HIVinfected individual without hepatitis $\mathrm{C}$ co-infection. Clin Nephrol 2002; 57 (2): 154.

8. Shahabdeen S, Fleszler FS, Falkowitz DC. Type I membranoproliferative glomerulonephritis (MPGN) in HIV without hepatitis C (HEP C) coinfection with rapid remission with steroid and angiotensin converting enzyme inhibitors (ACEI) without higly active anti retroviral therapy (HAART). Am J Kid Dis 2008; 51(4): A87 [Abstract]. 